\title{
Breaking up is hard to do: why disinvestment in medical technology is harder than investment
}

Marion Haas PhD, Professor, Centre for Health Economics Research and Evaluation, University of Technology

Jane Hall PhD, Professor, Centre for Health Economics Research and Evaluation, University of Technology

Rosalie Viney PhD, Associate Professor, Centre for Health Economics Research and Evaluation, University of Technology

Gisselle Gallego PhD, Adjunct Lecturer, 2Community Based Health Care Research Unit, Faculty of Health Sciences, University of Sydney,

Corresponding author. Email: marion.haas@chere.uts.edu.au

\begin{abstract}
Health care technology is a two-edged sword-it offers new and better treatment to a wider range of people and at the same time is a major driver of increasing costs in health systems. Many countries have developed sophisticated systems of Health Technology Assessment (HTA) to inform decisions about new investments in new health care interventions. In this paper we question whether HTA is also the appropriate framework for guiding or informing disinvestment decisions.
\end{abstract}

In exploring the issues related to disinvestment, we first discuss the various HTA frameworks which have been suggested as a means of encouraging or facilitating disinvestment. We then describe available means of identifying candidates for disinvestment (Comparative Effectiveness Research, clinical practice variations, clinical 
practice guidelines) and for implementing the disinvestment process (PBMA and related techniques).

In considering the possible reasons for the lack of progress in active disinvestment we suggest that HTA is not the right framework as disinvestment involves a different decision making context. The key to disinvestment is not just what to stop doing but how to make it happen- that is decision makers need to be aware of funding disincentives. 
Key Question Summary

What is known about this topic?

Disinvestment is an increasingly popular topic amongst academics and policy makers.

Most discussions focus on the need to increase disinvestment as a corollary of

investment, the lack of overt disinvestment decisions and the use of a framework based

on health technology assessment (HTA) to implement disinvestment.

What does this paper add?

This paper focuses on the difficulties associated with deciding which technologies to disinvest in and the problems in using an HTA framework to make such decisions when disinvestment involves a different decision making context from that of investment.

What are the implications for practitioners?

The key to disinvestment is not just what to stop doing but how to implement such decisions. Making it happen means being aware of funding disincentives. 


\section{Introduction}

Developments in health care technology, including drugs, diagnostic and procedural interventions, have had a major impact on the scope of health care, by expanding the range of people who can be offered treatment and the complexity of the treatments available. But health technology is also a major driver of rising health care costs. Thus, it not surprising that most attention has been directed toward developing a robust framework to evaluate new technologies proposed for introduction into health care practice. The development of formal health technology assessment (HTA) can be traced to the early 1970s and was stimulated in part by a concern to assure the safety and efficacy of new interventions, but also by concern about rising costs, and the need to ensure that new technologies represented good buys ${ }^{1}$. Although HTA encompasses many perspectives, including ethical, societal, and legal issues, its core focus is the evaluation of safety, efficacy and cost-effectiveness of alternative treatments or other procedures; and, at least for new and emerging technologies, to ensure they provide 'value for money'.

By focusing health technology assessment (HTA) efforts on new investment, many technologies in general use have never been appropriately evaluated, and their costeffectiveness is unknown. The early advocates of HTA argued that if new technologies were accepted for funding on the basis of cost-effectiveness, the armamentarium of 
funded technologies would, over time, become more cost-effective as the new (evaluated) technologies replaced the existing ones, thus improving the efficiency of the health system. However, as long as they remain safe and are not shown to do harm, existing technologies are generally not reviewed; thus their relative value for money is unknown.

In Australia, for example, although new technologies have been subject to formal HTA for over a decade, only about three percent of all technologies currently reimbursed have been subject to formal review ${ }^{2}$. In addition, many technologies are approved for reimbursement on a cost-minimisation basis, providing the same health outcome for the same cost as existing technologies. The implicit assumption is that there will be no additional cost from the new technology/procedure. However, in practice, these new treatments often become additional to rather than alternatives to the already funded treatments, creating pressure for additional spending.

The aim of this paper is to explore a number of issues related to disinvestment in light of recent discussion in the literature. Disinvestment refers to processes by which a health system or service removes technologies, without necessarily replacing them. First, we describe current approaches to encourage or facilitate disinvestment by applying existing HTA methods and processes. Second, we briefly discuss potential means of identifying candidate technologies for disinvestment; and some approaches to 
the implementation of a disinvestment process. Third, we discuss the possible reasons for the lack of progress and the challenges of designing a framework for disinvestment in health technologies.

\section{Disinvestment through Health Technology Assessment}

The term disinvestment is used with a range of meanings and there are different ways of examining this concept.

Elshaug et al defined it as "the process of withdrawing (partially or completely) health resources from any existing health care practices, procedures, technologies and pharmaceuticals that are deemed to deliver no or low health gain for their cost and thus [do] not [represent] efficient health resource allocation" ${ }^{\prime 3}$. Disinvestment has also been described as the cessation or restriction of potentially harmful, clinically ineffective or cost inefficient practices ${ }^{4}$. Even though Goodman does not define disinvestment, obsolete/outmoded/abandoned technologies are described as those that have been superseded or demonstrated to be ineffective or harmful ${ }^{5}$.

The disinvestment process could also be described as explicit or implicit. Person et al identified explicit disinvestment as the process of taking resources from one service in order to use them for other purposes (i.e. reallocation of resources) (Pearson and 
Littlejohns, 2007). "Implicit" disinvestment is best described as replacement/updating of practice and it occurs when a technology or intervention is superseded and therefore falls out of use. Definitions are diverse and demonstrate that apart from reasons of safety, there are no agreed operational criteria for disinvestment.

Several countries have formal HTA programs but, although there is a view that a similar approach can be used to implement disinvestment ${ }^{3,4}$, there is little evidence that active disinvestment is occurring ${ }^{6}$. In Australia, a recent review found that existing HTA processes are focused on the assessment of new technologies ${ }^{7}$. The operation of the Pharmaceutical Benefits Advisory Committee (PBAC), includes explicit criteria for removing a drug from the Pharmaceutical Benefits Scheme (PBS), but products de-listed to date have been largely as a result of new information becoming available about effectiveness and safety, or voluntary withdrawal by the manufacturer. Thus, any disinvestment that occurs in medicines in Australia tends to be implicit, resulting from the replacement in clinical use of current drugs with new, more effective drugs. Similarly, for the Medical Benefits Scheme (MBS), delisting has occurred through the Australian Department of Health and Ageing, generally as a result of an existing item falling into disuse. Although the review commented that "it will become increasingly difficult for governments to fund new health technologies unless they have the capacity 
to identify and remove funding for those that are less effective" none of the recommendations address how it might be achieved ${ }^{7}$.

In contrast to this, the UK National Institute for Health and Clinical Excellence (NICE) has a mandate "to identify and stop ineffective interventions and make health services more equitable across the country" ${ }^{\prime \prime}$. Strategies used include technology appraisals, clinical guidelines and commissioning guidelines. The last are web based guides designed to set benchmarks for service levels, and advice on issues such as local needs assessment and opportunities for disinvestment ${ }^{9}$. NICE has identified 102 interventions for which there is little or no evidence of benefit; 27 are covered by guidance, guidance is being developed for a further 30 and further assessment is being considered for $45^{10}$. As yet there is no clear evidence that disinvestment has been successfully implemented, which may be because the process relies on uptake of guidance at the health service level.

Germany provides a possible example of active disinvestment. After an evaluation by the Institute for Quality and Efficiency in Health Care (IQWiG), the Federal Joint Committee (FJC) drugs can be placed on a "negative" list and will not be reimbursed by the German statutory sickness funds. In Canada, the Ministry of Health and the Ontario Medical Association agreed to identify services "not medically necessary" in response to 1990s fiscal cuts. Candidate technologies were reviewed by an ad hoc commission of 
individuals representing the Ministry, the Medical Association, consumers, and academia, and several services were excluded or partially excluded from insurance $\operatorname{cover}^{10}$. However the "principles were not well articulated or publicized by the decision makers"11. A recent report by the Canadian Agency for Drugs and Technologies in Health (CADTH) discusses disinvestment, barriers to its implementation and some proposed approaches, but does not provide a description of what is currently happening in Canada or elsewhere ${ }^{11}$. The Danish Centre for Evaluation and Health Technology Assessment (DACEHTA) initiated a pilot project in 2004 to "assess improper use or potentially obsolete technologies" with a focus on imaging technologies. The rationale is not reported and the information available is limited to a conference abstract ${ }^{12}$. There have been two Spanish projects aimed at disinvestment ${ }^{10}$. Both involved developing a guide to identify and assess obsolete technologies, but little information has been published ${ }^{13}$.

This overview of processes across a range of countries demonstrates that disinvestment in cost-ineffective technologies as a component of HTA is still a rare, relatively slow process. The focus of formal health technology assessment and cost-effectiveness evaluation in most countries that have such formal processes is heavily weighted towards decisions about whether to invest in or fund new health technologies, hence favouring increased expenditure that is justified by increased health outcomes. In some systems the technology appraisal decision leads to an explicit decision to increase 
funding to adopt the technology (for example PBAC and MSAC in Australia), in other countries there may not be the same explicit decision, but there is rarely identification of which technologies within the existing budget will be defunded to allow the new technology to be adopted. In addition, there are two stages to disinvestmentidentifying cost-ineffective technologies, and implementing the disinvestment process. While the first may happen, the second does not automatically follow.

\section{Identifying candidate technologies for disinvestment}

Comparative Effectiveness Research (CER), research into clinical practice variations and clinical practice guidelines have been suggested as potential means of identifying candidate technologies for disinvestment. CER involves the systematic appraisal of the benefits and risks of alternative treatments and other health care interventions (e.g. screening $)^{14}$ and a new over-arching, public-private, co-ordinating agency, the Patient Outcomes Research Institute (PORI), has been established to oversee the program although its Charter specifically excludes any consideration of economic implications ${ }^{15-}$

18. The provision of information from evaluations is expected to change technology use by facilitating better decisions by clinicians as well as consumers, purchasers and policy makers. A list of priority topics for review has been developed by the Institute of Medicine $^{19}$. The development of the candidate topics was a major exercise in itself. 
Variations in the use of health services and per capita expenditure have been observed widely, over countries and over time, much of it unexplained by differences in population characteristics or without improved health outcomes ${ }^{20}$. The large literature covering this topic has been driven by a perceived need to identify causes of clinical variation amenable to intervention. So systematic investigations of practice variations may identify candidate technologies for disinvestment.

Clinical practice guidelines are designed, inter alia, to reduce the use of unnecessary, ineffective or harmful interventions ${ }^{21}$. Guidelines are most appropriately based on a process of systematic identification and synthesis of the best available evidence, as is CER. Guidelines in themselves are not a disinvestment strategy, as many recommend additional interventions. However, they should identify not just what should be done, but what should not be done, enabling candidate technologies to be identified for disinvestment.

\section{Implementing disinvestment}

Little attention has been given to implementing a disinvestment decision. When guidelines were first developed, it was assumed that the provision of evidence-based information would by itself change practice so that ineffective interventions would be replaced by those recommended in the guideline. But experience has shown that dissemination is not sufficient to ensure implementation, and that other strategies and 
incentives are required ${ }^{21}$. In addition, even interventions deemed ineffective or costineffective in one clinical context may be effective in another, and therefore should be available.

One approach to reallocating resources within a specific context is Program Budgeting and Marginal Analysis (PBMA), which has now been used for over 30 years in the health sector ${ }^{22}$. It involves a formal assessment of the costs and benefits of alternatives within a management context of planning and priority setting. Even in this context activities for investment are identified far more readily than are those for disinvestment. Similar approaches, such as ACE (Assessing Cost Effectiveness) Health Sector Wide Disease Based Model, Health Benefits Group/Health Resource Group, have been described $^{23-28}$.

Another approach to changing practice patterns is the use of pay for performance ${ }^{29}$. While ideally this would mean paying for improved health outcomes, the problem is that it is difficult to reward better outcomes directly, as there are many influences beyond medical care that affect final outcomes and considerable time between the medical intervention and the outcome. Thus in practice, incentives have been directed towards reinforcing appropriate care, such as in the UK Quality and Outcomes Framework, under which GPs are rewarded for achievement of nearly 200 specified indicators ${ }^{30}$ or various US pay for performance schemes ${ }^{31}$. Results available from the 
evaluation of schemes in this rapidly developing field of research indicate that such incentives may change practice ${ }^{32}$, results are mixed ${ }^{33,34}$ and these schemes are open to gaming $^{35}$. Another approach emphasises the use of performance reporting to enhance the role of consumers in choosing their own health insurance, in managed competition markets, or their choice of clinician, hospital and/or treatment ${ }^{33}$. New organizational structures use various forms of budget holding and blended payments to enhance the purchasing or commissioning of appropriate treatment, including the efficient use of technologies ${ }^{36,37}$.

\section{Challenges to implementing a program of active disinvestment}

So far, disinvestment has been seen as a corollary of investment. However, the questions relating to investment and disinvestment are fundamentally different. Other reviews of this topic conclude that lack of resources for research into established technologies, inadequate resources and lack of political, clinical and administrative will to support the process are factors which impede implementation ${ }^{4,24}$. Although these may be significant barriers, a major challenge is to identify which technologies are candidates for disinvestment. Even in the reported pilot studies, there is little description of the actual process used to identify technologies for disinvestment; and in the US, the CER program involved a major review exercise to identify candidate technologies and there are currently more than 100 topics on the priority list. 
An understanding of incentives helps to explain why this is so. In the case of new technologies, there is a clear incentive for initiating an appraisal, particularly when the HTA process is linked to funding and/or reimbursement. For existing technologies, unless there is new evidence of positive harm, there is little to gain in removing access. There is an option value for clinicians and patients in having the technology available and funded. Similarly, the use of PBMA relies on managers and clinicians to identify areas of disinvestment; they will have no incentive to do so if the freed resources will not be available to fund alternative interventions or services in the same program.

\section{Implications for health services decision makers}

The key to disinvestment is not just what technologies to cease funding but how to create the right environment for this to occur- in other words, how to establish and implement disincentives to use technologies it is considered desirable to cease using. It is important to recognise that an active program or strategy of disinvestment will create losses, to clinicians, to consumers and to providers of the technology. At the same time, the additional benefits and/or savings from any disinvestments may not be realised for a considerable period of time and there is a risk that, for some products, interventions or services, cost savings, in particular, may not be realised. Moreover, the gains from disinvestment are likely to be more diffuse and less readily specified than any losses. Hence losers have a stronger incentive to lobby for the continuation of the status quo, 
than gainers do for effecting the change. In short, linking HTA to reimbursement decisions provides a strong incentive for new technologies to be identified and subject to appraisal. There are disincentives for disinvestment, and the process of identifying the target technologies is made more complex by this.

Because removing resources is easier than providing additional resources, the threshold for disinvestment may need to be different from the threshold for investment. Further, if decision makers are serious about disinvestment, they may also need to be more stringent in deciding what level of evidence and what level of cost-effectiveness is acceptable for funding a new technology. That is, the challenges associated with disinvestment bring additional challenges for the standards applied to investment decisions.

Our conclusion is that the impetus for disinvestment relies on changing practice and such change can only come from changing incentives. These might apply to clinicians, but also to consumers and funders. The continued debate about disinvestment must recognise the role of incentives, and future work should evaluate how changing incentives affects technology use and disuse. 


\section{References}

1. Banta D. What is technology assessment? International Journal of Technology Assessment in Health Care. 2009. Supp 1: 7-9.

2. Medical Benefits Reviews Task Group, Department of Health and Ageing. Development of a Quality Framework for the Medicare Benefits Schedule: Discussion Paper. April 2010. Australian Government.

3. Elshaug AG, Hiller JE, Tunis SR, Moss JR. Challenges in Australian policy processes for disinvestment from existing, ineffective health care practices. Australia and New Zealand Health Policy 2007 4: 23.

4. Ibargoyen-Roteta N, Gutierrez-Ibarluzea I, Asua J, Benguria-Arrate G, GalnaresCordero L. Scanning the horizon of obsolete technologies: Possible sources for their identification. International Journal of Technology Assessment in Health Care 2009 25: 249-254.

5. Goodman C. 2004. HTA 101 introduction to health technology assessment. Lewin Group: Falls Church, Va.

6. Kanavos P, Persson U, Drummond M. 2008. The future of health technology assessment in Europe. London School of Economics: London.

7. Australian Government, Department of Health and Ageing. Review of Health Technology Assessment in Australia. Commonwealth of Australia, 2009. 
8. Pearson S, Littlejohns P. 2007. Reallocating resources: How should the National institute for health and clinical excellence guide disinvestment efforts in the National Health Service? Journal of Health Services Research and Policy 12: 160-165.

9. Hailey D. HTA in Australia. International Journal of Technology Assessment in Health Care. 2009 Supp 1. 61-67.

10. Hughes D, Ferner R. New drugs for old: disinvestment and NICE. British Medical Journal 2010 340:c572.

11. Giacomini M. The which-hunt: Assembling Health Technologies for Assessment and Rationing. Journal of Health Politics, Policy and Law 1999 24:715-758.

12. Frellsen MB, Kristensen FB. Technologies that are claimed useless or applied in a useless way should undergo HTA and be discarded from daily practice if proven so. Case: Routinely performed chest x-ray at admission. Italian Journal of Public Health 2005 2: 65 .

13. Pritec tool for obsolete health technologies. http://www.pritectools.es/Controlador/documentosAction.php

[Accessed 20 August 2009].

14. Congress of the United States Congressional Budget Office. Research on the comparative effectiveness of medical treatments: Issues and options for an expanded federal 
role. Pub. No. 2975. Congress of the United States Congressional Budget Office:

Washington, 2007.

15. Academy Health. Incorporating costs into comparative effectiveness research. Academy Health: Washington, 2009.

16. Institute of Medicine. Knowing what works: A roadmap for the nation. National Academies Press: Washington, 2009.

17. Clancy C, Collins F. S. Patient-Centered Outcomes Research Institute: The intersection of science and health care. Sci. Transl. Med. 2010 2, 37cm18.

18. Sox HC, Comparative Effectiveness Research: a progress report. Annals of Internal Medicine. 2010; 153:469-472.

19. Institute of Medicine Initial National Priorities for Comparative Effectiveness Research. The National Academies Press, Washington, USA, 2009.

20. Hall J. Variations in health care costs and utilisation. NSW Treasury: 2008.

21. NHMRC. A guide to the development, implementation and evaluation of clinical practice guidelines. Endorsed 1998. Commonwealth of Australia, 1999.

22. Mitton C, Peacock S, Donaldson C, Bate A. Using PBMA in health care priority setting: Description, challenges and experience. Applied Health Economics and Health Policy 2003 2: 121-127.

23. Carter, R., T. Vos, Moodie M, Haby M, Magnus A, Mihalopoulos C. Priority setting in health: Origins, description and application of the assessing cost effectiveness 
(ACE) initiative. Expert Review in Pharmacoeconomics and Outcomes Research 2008 8:

593-617

24. Segal, L. and D. Mortimer. A population-based model for priority setting across the care continuum and across modalities. Cost Effectiveness and Resource Allocation 2006 4: 6 .

25. Segal, L. and Y. Chen. Priority setting models for health: The role for priority setting and a critique of alternative models. A summary. Centre for Health Program Evaluation Working Paper 119, 2001.

26. Eager K, Garrett P, Lin V. Health Planning: Australian perspectives. Sydney: Allen and Unwin, 2001

27. Segal, L., S. Day, et al. Priority Setting in Osteoarthritis. Research Paper 12. Melbourne Health Economics Unit, Monash University, 2006.

28. Segal, L., S. E. Day, et al. Can we reduce disease burden from osteoarthritis? Medical Journal of Australia 2004 180: S11-7.

29. Committee on Redesigning Health Insurance, P. M., Payment and Performance Improvement Programs, Institute of Medicine (2007). Rewarding Provider Performance: Aligning Incentives in Medicare Washington DC, National Academies Press.

30. Department of Health., Using the Commissioning for Quality and Innovation (CQUIN) payment framework. 2008, Department of Health. London. 
31. Rosenthal MB, Fernandopulle R, Song HR, and Landon BE. Paying for Quality: Providers' Incentives for Quality Improvement, Health Affairs, 23 (2):127-41, MarchApril, 2004.

32. Duckett S, Daniels S, Kamp M, Stockwell A, Walker G, Ward M. Pay for performance in Australia: Queensland's new Clinical Practice Improvement Payment J Health Serv Res Policy 2008;13:174-177

33. Dudley, R.A., et al., Strategies to support quality-based purchasing: A review of the evidence. AHRQ Publication No. 04-0057. 2004, Rockville: Agency fof Healthcare Research \& Quality.

34. Mullen KJ, Frank RG, Rosenthal MB. Can you get what you pay for? Pay-for-performance and the quality of healthcare providers. RAND Journal of Economics, 2010 41, 64-91

35. Gravelle H, Sutton M, Ma A. Doctor behaviour under a pay for performance contract: treating, cheating and case finding? Economic Journal 2010;120:F129-F56.

36. Hall J. Medicare Select: A bold reform? The Australian Economic Review, 2010 vol. 43, no. 1, pp. 63-70.

37. Shortell SM, Casalino LP, Fisher ES. How the Center for Medicare and Medicaid Innovation Should Test Accountable Care Organizations Health Affairs 29, no. 7 (2010): 1293-98. 